\title{
At the Cutting Edge
}

\section{Dear Reader,}

This one hundredth issue of ATZelectronics offers an occasion to reflect briefly on what we have covered so far. While all areas of vehicle development are naturally subject to dynamic processes, no area has developed as rapidly as that of automotive electronics and electric systems. As we take a look back, we find that while attention has indeed shifted away from some topics, others remain highly relevant, although perhaps at a different level.

The first issue we published in March 2006 - Germany was preparing at the time to host what would turn out to be a successful FIFA World Cup - included two still remarkable articles on the topic of ADAS: The article by Robert Knoll (Bosch) explained the "clear superiority of NIR technology" for enhanced night vision in vehicles. Markus Schupfner and Karl Artmann (Siemens) described the use of electronic horizontal situation indicators in navigations systems to optimize route calculation. The topic, which included a discussion of fuel economy, is currently considered to be highly relevant.

Other articles in the first issue addressed flexible control devices for injection actuation and dynamic software-module testing as a means of optimizing embedded systems and enabling early error detection. These topics continue to concern us today, and the articles offer a good overview of early development from today's perspective. Indeed, while turning the pages of the last 14 years of ATZelectronics, readers will find no subject that is not still relevant today, although the relevance is often at a different level of development. Of less concern are only various discussions of classic mechatronics.
Over the years, ATZelectronics has occasionally shifted its focus in an effort to pursue the latest developments. In addition to key topics such as automated driving, E/E architectures, embedded systems and semiconductors, we have devoted much attention in recent years to electric vehicles and network infrastructure. This shift led to some overlap with ATZ and MTZ. Since the beginning of last year, ATZelectronics has recalibrated its focus and returned to topics that reflect its core as a brand, including vehicle electronics and electric systems. This dynamic area plays to our strengths. It is where we are always at the cutting edge.

We hope you enjoy reading this latest issue of ATZelectronics!

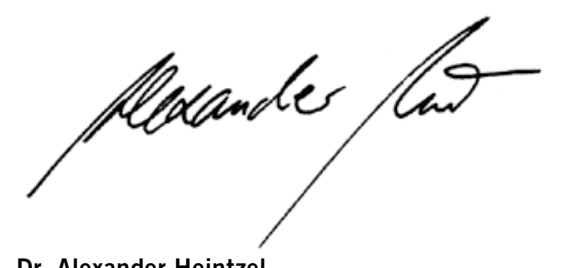

Dr. Alexander Heintzel

Editor in Chief

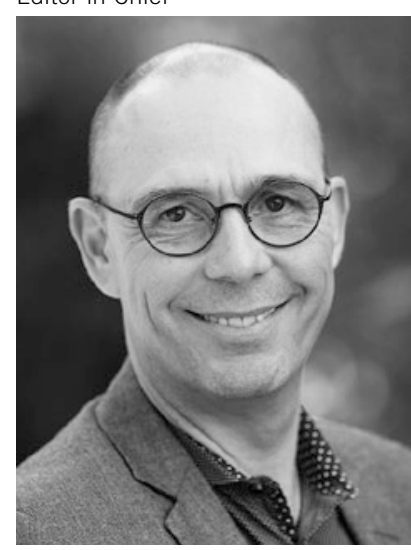

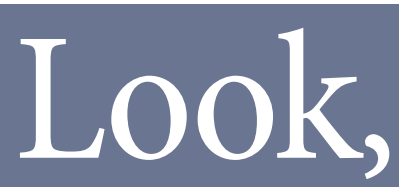

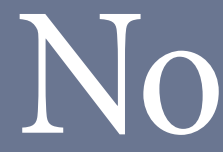

Hands!

Just a matter of the right methods.

Driver assistance systems are changing the very nature of cars. Connected and increasingly open, these security-critical assistants can shake the very foundations of conventional wisdom. We strike the right balance and put it all on a solid footing by combining systems engineering, safety, security and smart testing methods. It all comes together in one unified function-driven concept. And our solutions are scalable and reusable to give you even more avenues to explore.

ITK Engineering GmbH - Take the open road to driving freedom.

www.itk-engineering.com

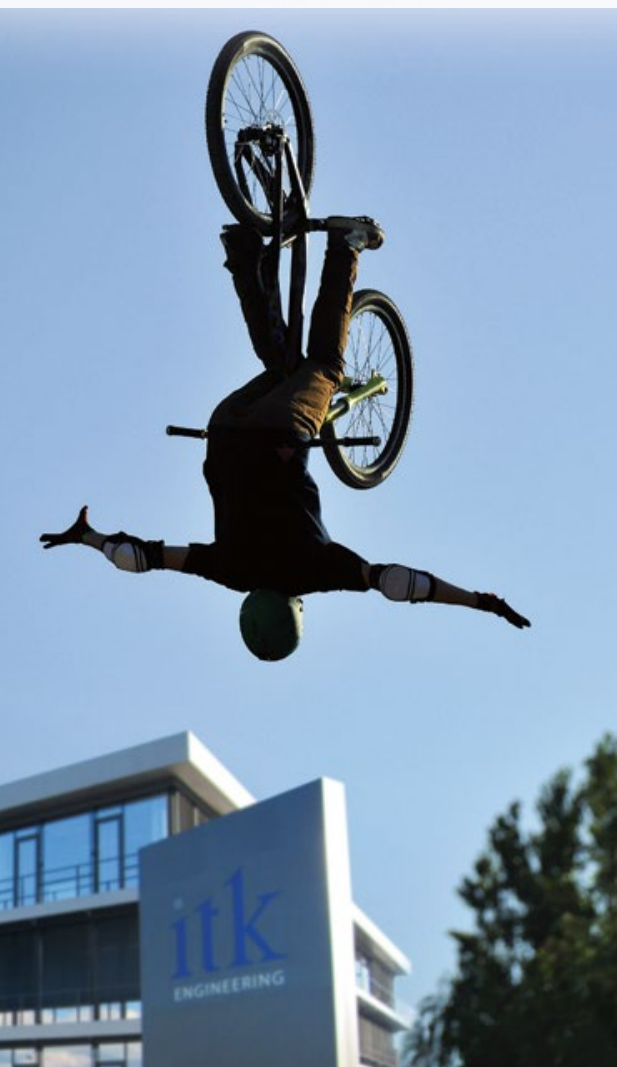

